\title{
Switching Surface Design for Periodically Operated Discretely Controlled Continuous Systems
}

\author{
Axel Schild and Jan Lunze \\ Institute of Automation and Computer Control, Ruhr-Universitaet Bochum, \\ Universitaetsstrasse 150, 44780 Bochum, Germany \\ \{Schild, Lunze\}@atp.rub.de
}

\begin{abstract}
Discretely controlled continuous systems (DCCS) represent an important class of hybrid systems, in which a continuous process is regulated by a discrete controller. The paper introduces a novel modelbased design procedure for periodically operated DCCS with the objective to produce a periodic stationary operation. The method exploits an equivalence to periodic control systems to obtain an event-driven switching strategy that locally stabilizes a predetermined limit cycle and enforces a desired transient behavior. In contrast to earlier results, the controller responds to deviations without a dead time.
\end{abstract}

\section{Introduction}

Discretely controlled continuous systems (DCCS) have recently received much attention throughout the hybrid systems community $[1,2,3,4,5,6,7]$. Such systems form a control loop composed of a continuous plant and a discrete-event controller (Fig. 1(a)). This structure is found in many application domains, such as power electronics, manufacturing systems, process engineering, mechanics and robotics. The control task of DCCS is to switch the plant's mode of operation at opportune moments to meet specifications defined in terms of the continuous variables at stationary operation. As a central characteristic, the working principle of these systems demands a never ending switching action, which prevents the continuous state trajectory to converge towards an equilibrium state. Instead, a periodic or even a chaotic stationary motion is observed.

This paper presents a novel model-based design procedure for the event generator included in the discrete controller. The presented approach produces static

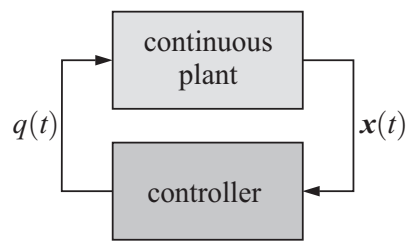

(a)

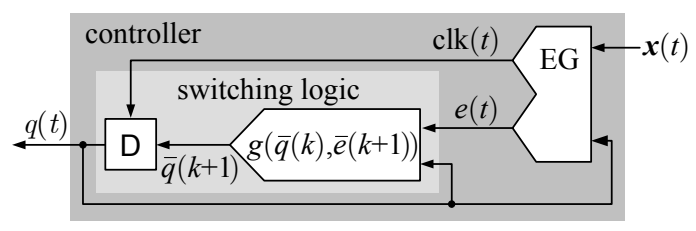

(b)

Fig. 1. Structure of a DCCS: (a) control loop, (b) discrete controller. 
switching planes and allows to influence the local loop properties systematically. Compared to previously published approaches, the discrete controller is of lower complexity and its control actions are instantaneously applied without any delay. The method does not impose any restrictions on the number of continuous states or the number of operation modes. Furthermore, it allows for a partial design, if a subset of system-inherent switching surface must not be altered.

The paper is organized as follows: Section 2 recalls the model of a DCCS. Following the problem formulation (Sect. 3), all design steps are presented in Sect. 4. The key idea is to recast the original problem into a periodic linear control problem (Sect. 4.1). With the equivalence stated in Theorem 1, novel results for checking the local stabilizability of a DCCS along a closed orbit are derived (Sect. 4.2) and summarized in Theorem 3. Section 4.3 classifies modes as effective or ineffective, which is crucial for obtaining feasible design results. Section 4.4 summarizes the design algorithm, which is successfully applied to a laboratory plant in Sect. 5. The experimental data demonstrate the excellent loop performance, which is attained by this discrete controller.

Literature. In the past, research work on DCCS primarily focused on analysis methods, whereas model-based design approaches have only been scarcely developed. Recently, the optimal start-up of DCCS under known initial conditions was addressed in $[8,9]$ and extended to unknown initial states in $[10,11]$. Lyapunov-based switching with application to switching power converters was discussed in $[12,13]$. The primary objective considered in these publications is to drive the continuous trajectory into a neighborhood of a desired terminal state.

The problem of stabilizing limit cycles of smooth nonlinear systems, on the other hand, has been investigated over the past two decades starting with [14]. It was extended to non-smooth dynamical systems in [15], where the authors advocate the use of switching and state-resetting to affect the stability of recurrent motions. The question of how to systematically design the "control law" in case of multiple control interactions over one period remained unanswered, until a model-based solution was presented in [16]. The approach proposed therein relies on the dynamic adjustment of nominal switching surfaces by means of a switching surface controller (SSC). Because the resulting controller responds to deviations with a potentially large delay, the loop performance may be unsatisfactory in the presence of disturbances. Moreover, the approach assumes the knowledge of nominal switching planes, which have a strong influence on the amplitude of the control actions issued at runtime. Therefore, an important aspect is to determine the best possible nominal switching plane orientations with respect to the control objective, which is addressed in this paper.

\section{Modeling of periodically operated discretely controlled continuous systems}

\subsection{Hybrid model}

The main contribution of this paper is a novel model-based design method for the event generator of periodically operated discretely controlled continuous systems 
(DCCS), i.e. systems that recurrently execute a predetermined sequence

$$
Q_{\mathrm{LC}}=\left(\bar{q}_{0}^{\star} \bar{q}_{1}^{\star} \ldots \bar{q}_{p-1}^{\star}\right)
$$

of $p$ distinct operation modes $\bar{q}_{i}^{\star} \neq \bar{q}_{j}^{\star}$. This section summarizes a model, which reflects all relevant aspects of the closed-loop behavior and represents a tailored version of the general hybrid model introduced in $[16,17]$.

The model consists of three components: a continuous plant, an event generator and a discrete switching logic (Fig. 1(b)). The continuous dynamics are governed by the state equation

$$
\dot{\boldsymbol{x}}(t)=\boldsymbol{f}(\boldsymbol{x}(t), q(t)), \boldsymbol{x}(0)=\boldsymbol{x}_{0},
$$

where $\boldsymbol{x} \in \mathbb{R}^{n}$ is the continuous state. The plant's sole accessible input $q(t)$ is restricted to the finite set $\mathcal{Q}=\left\{\bar{q}_{0}^{\star}, \ldots, \bar{q}_{p-1}^{\star}\right\}$ of operation modes. For each mode $\bar{q}_{k}^{\star}$, the continuous dynamics (2) possess a different equilibrium state.

The event generator implements a piecewise affine event function

$$
\Phi(\boldsymbol{x}, q)=\boldsymbol{c}^{\mathrm{T}}(q) \boldsymbol{x}(t)-d(q),
$$

which implicitly defines a switching hyperplane

$$
\mathcal{S}(q)=\{\boldsymbol{x}: \Phi(\boldsymbol{x}, q)=0\}
$$

for each mode $q$. The vector $\boldsymbol{c}^{\mathrm{T}}(q)$ and the scalar $d(q)$ determine a plane's orientation and its location in the state space. The event generator outputs a trigger signal

$$
\operatorname{clk}(t)= \begin{cases}0, & \text { if }|\Phi(\boldsymbol{x}(t), q(t))|>0 \\ 1, & \text { if } \Phi(\boldsymbol{x}(t), q(t))=0\end{cases}
$$

which initiates an update of the memory (D) according to

$$
\bar{q}(k+1)=q\left(\bar{t}(k+1)^{+}\right)=\bar{q}_{((k+1) \bmod p)}^{\star}, \quad \bar{q}(0)=\bar{q}_{0}^{\star}
$$

at the switching time

$$
\bar{t}(k+1)=\min _{t>\bar{t}(k)} t: \operatorname{clk}(t)=1
$$

Here $\bar{t}(k)^{+}$denotes the limit from above. As the DCCS is assumed to execute the predetermined mode sequence $Q_{\mathrm{LC}}$, the second output $e(t)$ of the event generator (Fig. 1) can be omitted. In the following, signals

$$
\overline{\boldsymbol{x}}(k)=\boldsymbol{x}(\bar{t}(k)), \bar{q}(k)=q\left(\bar{t}(k)^{+}\right)
$$

sampled at switching instants are indicated by a bar and enumerated by a counter $k$. The time span

$$
\bar{\tau}(k)=\bar{t}(k+1)-\bar{t}(k)>0
$$


is called the activation duration of mode $\bar{q}(k)$ and $\tau$ denotes the elapsed time since the last switching. A DCCS execution over $N$ switchings is denoted by

$$
\chi\left(\boldsymbol{x}_{0}^{h}, t_{0}, N\right)=(\boldsymbol{x}(t), q(t), \mathcal{T}(N)), \quad \boldsymbol{x}_{0}^{h}=\left(\boldsymbol{x}_{0} \bar{q}_{0}^{\star}\right)^{\mathrm{T}}, t \in\left[t_{0}, \bar{t}(N)\right]
$$

with $\mathcal{T}(N)=\left(\left(\left[t_{0}, \bar{t}(0)\right],(\bar{t}(0), \bar{t}(1)], \ldots\right)\right.$ being a finite sequence of $N$ activation intervals. The continuous state evolution starting in $\left(\begin{array}{ll}\boldsymbol{x}_{0} & \bar{q}_{0}^{\star}\end{array}\right)^{\mathrm{T}}$ is referred to as $\boldsymbol{x}\left(\tau, \boldsymbol{x}_{0}, \bar{q}_{0}^{\star}\right)$. Executions $\chi^{\star}\left(\boldsymbol{x}_{0}^{h, \star}, 0, N\right)$ that satisfy

$$
\begin{aligned}
\bar{q}^{\star}(k+p) & =\bar{q}^{\star}(k) \\
\boldsymbol{x}^{\star}\left(\tau, \overline{\boldsymbol{x}}^{\star}(k+p), \bar{q}^{\star}(k+p)\right) & =\boldsymbol{x}^{\star}\left(\tau, \overline{\boldsymbol{x}}^{\star}(k), \bar{q}^{\star}(k)\right), \quad \forall \tau \in\left(0, \bar{\tau}^{\star}(k)\right]
\end{aligned}
$$

for all $k \leq N-p$ are called periodic of order $p$ and are indicated by a star. The corresponding closed orbit $\mathcal{L}_{\mathrm{LC}}$ traced out by $\chi^{\star}\left(\boldsymbol{x}_{0}^{h, \star}, 0, p\right)$ is called the limit cycle. For notational convenience, let $\overline{\boldsymbol{x}}^{\star}\left(\bar{q}_{k}^{\star}\right)$ denote the switch point $\overline{\boldsymbol{x}}^{\star}(k) \in$ $\mathcal{L}_{\mathrm{LC}}$, at which the mode $\bar{q}^{\star}(k)=\bar{q}_{k}^{\star}$ is activated for the next $\bar{\tau}^{\star}\left(\bar{q}_{k}^{\star}\right)$ time units.

Remark 1. Any periodic execution $\chi^{\star}\left(\boldsymbol{x}_{0}^{h}, 0, p\right)$ satisfies

$$
\min _{\tau \in\left[0, \bar{\tau}^{\star}\left(\bar{q}_{k}^{\star}\right)\right)}\left|\boldsymbol{c}^{\mathrm{T}}\left(\bar{q}_{k}^{\star}\right)\left(\boldsymbol{x}^{\star}\left(\tau, \overline{\boldsymbol{x}}^{\star}\left(\bar{q}_{k}^{\star}\right), \bar{q}_{k}^{\star}\right)-\overline{\boldsymbol{x}}^{\star}\left(\bar{q}_{k+1}^{\star}\right)\right)\right|>0
$$

for all $k=0 \ldots(p-1)$, since the first intersection of $\boldsymbol{x}^{\star}\left(\tau, \overline{\boldsymbol{x}}^{\star}\left(\bar{q}_{k}^{\star}\right), \bar{q}_{k}^{\star}\right)$ and the planes $\mathcal{S}\left(\bar{q}_{k}^{\star}\right)$ must occur at $\overline{\boldsymbol{x}}^{\star}\left(\bar{q}_{k+1}^{\star}\right)$. Accordingly, (7) constitute critical constraints, if the event function (3) is not given but must be synthesized to enforce a particular periodic execution.

\subsection{Sampled data model}

Sampling an execution (6) at switching instants yields the sampled execution

$$
\bar{\chi}\left(\boldsymbol{x}_{0}^{h}, t_{0}, N\right)=\left((\overline{\boldsymbol{x}}(0) \bar{q}(0) \bar{t}(0))^{\mathrm{T}}, \ldots,(\overline{\boldsymbol{x}}(N) \bar{q}(N) \bar{t}(N))^{\mathrm{T}}\right),
$$

which is obtained by $N$ iterated applications of the system's embedded map [18]. To synthesize switching planes (4) by means of a model, an analytic expression of this map's continuous component

$$
\overline{\boldsymbol{x}}(k+1)=\boldsymbol{H}_{x}(\overline{\boldsymbol{x}}(k), \bar{q}(k), \bar{\tau}(k))
$$

is needed. Unfortunately, a closed form representation of $\boldsymbol{H}_{x}$ is only possible for very simple DCCS. Concerning the vicinity of a stationary periodic execution $\chi^{\star}\left(\boldsymbol{x}_{0}^{h, \star}, 0, p\right)$ that transversally intersects with all switching planes, i.e.

$$
\left|\boldsymbol{c}^{\mathrm{T}}\left(\bar{q}_{k}^{\star}\right) \boldsymbol{f}\left(\overline{\boldsymbol{x}}^{\star}\left(\bar{q}_{k+1}^{\star}\right), \bar{q}_{k}^{\star}\right)\right|>0, \forall \bar{q}_{k}^{\star} \in \mathcal{Q},
$$

at least a linear approximation of $(9)$ for $\bar{q}(k)=\bar{q}_{k}^{\star}$ can be obtained as [19]

$$
\begin{aligned}
\delta \overline{\boldsymbol{x}}(k+1) & =\frac{\mathrm{d} \boldsymbol{H}_{x}}{\mathrm{~d} \boldsymbol{x}}\left(\overline{\boldsymbol{x}}^{\star}\left(\bar{q}_{k}^{\star}\right), \bar{q}_{k}^{\star}, \bar{\tau}^{\star}\left(\bar{q}_{k}^{\star}\right)\right) \delta \overline{\boldsymbol{x}}(k) \\
& =\left(\boldsymbol{I}-\frac{\boldsymbol{f}\left(\overline{\boldsymbol{x}}^{\star}\left(\bar{q}_{k+1}^{\star}\right), \bar{q}_{k}^{\star}\right) \boldsymbol{c}^{\mathrm{T}}\left(\bar{q}_{k}^{\star}\right)}{\boldsymbol{c}^{\mathrm{T}}\left(\bar{q}_{k}^{\star}\right) \boldsymbol{f}\left(\overline{\boldsymbol{x}}^{\star}\left(\bar{q}_{k+1}^{\star}\right), \bar{q}_{k}^{\star}\right)}\right) \frac{\partial \overline{\boldsymbol{x}}^{\star}\left(\bar{q}_{k+1}^{\star}\right)}{\partial \overline{\boldsymbol{x}}^{\star}\left(\bar{q}_{k}^{\star}\right)} \delta \overline{\boldsymbol{x}}(k) .
\end{aligned}
$$


Here, $\partial \overline{\boldsymbol{x}}^{\star}\left(\bar{q}_{k+1}^{\star}\right) / \partial \overline{\boldsymbol{x}}^{\star}\left(\bar{q}_{k}^{\star}\right)$ is the fundamental matrix of (2) for $q=\bar{q}_{k}^{\star}$ and $\boldsymbol{x}(0)=\overline{\boldsymbol{x}}^{\star}\left(\bar{q}_{k}^{\star}\right)$ at $t=\bar{\tau}^{\star}\left(\bar{q}_{k}^{\star}\right)$. The difference $\delta \overline{\boldsymbol{x}}(k)=\overline{\boldsymbol{x}}(k)-\overline{\boldsymbol{x}}^{\star}\left(\bar{q}_{k}^{\star}\right)$ denotes the sampled deviation of $\boldsymbol{x}(t)$ from the stationary periodic execution. Clearly, the approximation (11) is only well defined, if the transversality condition (10) holds.

A composition of (9) over a complete cycle $Q_{\mathrm{LC}}$ yields a first return map

$$
\overline{\boldsymbol{x}}(c+1)=\boldsymbol{P}_{x}(\overline{\boldsymbol{x}}(c), \bar{q}(c), \bar{\tau}(c))=\boldsymbol{H}_{x} \circ \ldots \circ \boldsymbol{H}_{x}(\overline{\boldsymbol{x}}(c), \bar{q}(c), \bar{\tau}(c)),
$$

which describes the evolution $\overline{\boldsymbol{x}}(c)$ of the switch points in the switching plane $\mathcal{S}(\bar{q}(c))$ associated to mode $\bar{q}(c+1)=\bar{q}(c)$. The counter $c$ is used to enumerate the executed cycles. Similar to (12), the linearized return map

$$
\begin{aligned}
\delta \overline{\boldsymbol{x}}(c+1) & =\frac{\mathrm{d} \boldsymbol{P}_{x}}{\mathrm{~d} \boldsymbol{x}}\left(\overline{\boldsymbol{x}}^{\star}\left(\bar{q}_{0}^{\star}\right), \bar{q}_{0}^{\star}, \bar{\tau}^{\star}\left(\bar{q}_{0}^{\star}\right)\right) \delta \overline{\boldsymbol{x}}(c) \\
& =\left(\prod_{k=0}^{p-1} \frac{\mathrm{d} \boldsymbol{H}_{x}}{\mathrm{~d} \boldsymbol{x}}\left(\overline{\boldsymbol{x}}^{\star}\left(\bar{q}_{k}^{\star}\right), \bar{q}_{k}^{\star}, \bar{\tau}^{\star}\left(\bar{q}_{k}^{\star}\right)\right)\right) \delta \overline{\boldsymbol{x}}(c)
\end{aligned}
$$

is obtained by composing (11) over a complete cycle $Q_{\mathrm{LC}}$, in this case starting at $\bar{q}_{0}^{\star}$. Note that (13) represents a linear autonomous discrete-time periodic system, which carries information about the local orbital stability of $\boldsymbol{x}^{\star}(t)$ [17].

Remark 2. In case of a piecewise constant vector field $\boldsymbol{f}(\boldsymbol{x}, q)=\boldsymbol{b}(q)$, the expressions (11) and (13) describe $\boldsymbol{H}_{x}$ and $\boldsymbol{P}_{x}$ exactly [5].

Remark 3. Assuming a transversal intersection of $\chi^{\star}\left(\boldsymbol{x}_{0}^{h, \star}, 0, p\right)$ with all switching planes $\mathcal{S}\left(\bar{q}_{k}^{\star}\right)$, the map $\boldsymbol{H}_{x}$ is a local $C^{1}$-diffeomorphism in a neighborhood of all $\overline{\boldsymbol{x}}^{\star}\left(\bar{q}_{k}^{\star}\right)$ [19]. As $\boldsymbol{P}_{x}$ results from the composition of $\boldsymbol{H}_{x}$ over a switching cycle, it is a local $C^{1}$-diffeomorphism in a neighborhood of $\overline{\boldsymbol{x}}^{\star}\left(\bar{q}_{0}^{\star}\right)$ as well.

\section{Problem formulation}

Problem 1. Consider a continuous plant (2), a switching logic (5) that cyclically generates the mode sequence $Q_{\mathrm{LC}}(1)$ and a predetermined stationary periodic execution $\chi^{\star}\left(\boldsymbol{x}_{0}^{h, \star}, 0, p\right)$ starting in $\boldsymbol{x}_{0}^{h, \star}=\left(\overline{\boldsymbol{x}}^{\star}\left(\bar{q}_{0}^{\star}\right) \bar{q}_{0}^{\star}\right)^{\mathrm{T}}$. The task considered in this paper is to find an event function (3) that renders the limit cycle $\mathcal{L}_{\mathrm{LC}}$ associated to $\chi^{\star}\left(\boldsymbol{x}_{0}^{h, \star}, 0, p\right)$ locally orbitally stable [16].

According to Prob. 1, the task is to find switching planes (4) that implicitly parameterize the activation duration $\bar{\tau}(\bar{q}(k), \delta \boldsymbol{x}(\tau))=\bar{\tau}^{\star}(\bar{q}(k))+\delta \bar{\tau}(\bar{q}(k), \delta \boldsymbol{x}(\tau))$ in terms of the mode and the deviation $\delta \boldsymbol{x}(\tau)=\operatorname{dist}\left(\boldsymbol{x}(\tau, \overline{\boldsymbol{x}}(k), \bar{q}(k)), \mathcal{L}_{\mathrm{LC}}\right)$ and thereby assure local orbital stability of $\mathcal{L}_{\mathrm{LC}}$. The latter implies that all multipliers

$$
m_{i}=\lambda_{i}\left(\prod_{k=0}^{p-1} \frac{\mathrm{d} \boldsymbol{H}_{x}}{\mathrm{~d} \boldsymbol{x}}\left(\overline{\boldsymbol{x}}^{\star}\left(\bar{q}_{k}^{\star}\right), \bar{q}_{k}^{\star}, \bar{\tau}^{\star}\left(\bar{q}_{k}^{\star}\right)\right)\right)
$$

of the return map's Jacobian (13) must lie inside the unit circle. 


\section{Switching Surface Design}

\subsection{Equivalent discrete-time periodic linear system}

According to (11), the event generator design concentrates on finding vectors $c^{\mathrm{T}}\left(\bar{q}_{k}^{\star}\right)$ that ensure orbital stability of $\mathcal{L}_{\mathrm{LC}}$. The sequel shows that by solving a classical linear periodic control problem, a set of feasible vectors $c^{\mathrm{T}}\left(\bar{q}_{k}^{\star}\right)$ for $k=1 \ldots(p-1)$ is obtained, which result in multipliers (14) satisfying $\left|m_{i}\right|<1$.

In the first step, the denominator of (11) is removed, by which the normals $c^{\mathrm{T}}\left(\bar{q}_{k}^{\star}\right)$ enter the linearized return map (13) nonlinearly. This nonlinear dependence vanishes, iff the design procedure generates vectors $c^{\mathrm{T}}\left(\bar{q}_{k}^{\star}\right)$ that satisfy

$$
\boldsymbol{c}^{\mathrm{T}}\left(\bar{q}_{k}^{\star}\right) \boldsymbol{f}\left(\overline{\boldsymbol{x}}^{\star}\left(\bar{q}_{k+1}^{\star}\right), \bar{q}_{k}^{\star}\right)=1, \forall \bar{q}_{k}^{\star} \in \mathcal{Q}
$$

The following Lemma translates the constraints (15) into equivalent conditions that can be explicitly accounted for in the design.

Lemma 1. A vector $\boldsymbol{c}^{\mathrm{T}}\left(\bar{q}_{k}^{\star}\right)$ satisfies the constraint (15), iff

$$
\operatorname{det}\left(\boldsymbol{I}-\boldsymbol{f}\left(\overline{\boldsymbol{x}}^{\star}\left(\bar{q}_{k+1}^{\star}\right), \bar{q}_{k}^{\star}\right) \boldsymbol{c}^{\mathrm{T}}\left(\bar{q}_{k}^{\star}\right)\right)=0
$$

Proof. The equivalence of (15) and (16) readily follows from the matrix determinant lemma [20], which says that $\operatorname{det}\left(\boldsymbol{I}-\boldsymbol{a} \boldsymbol{b}^{\mathrm{T}}\right)=1-\boldsymbol{a}^{\mathrm{T}} \boldsymbol{b}$.

With Lemma 1, a key result of the paper follows. Recall that two periodic linear system $\Sigma_{i}=\left\{\boldsymbol{A}_{i}(k), \boldsymbol{b}_{i}(k)\right\}, i=1,2$ are equivalent, if their monodromy matrices $\boldsymbol{\Psi}_{i}(p, 0)=\prod_{k=0}^{p-1} \boldsymbol{A}_{i}(k)$ are similar.

Theorem 1. The p-periodic linear system $\Sigma=\left\{\boldsymbol{A}_{d}(k), \boldsymbol{b}_{d}(k)\right\}$ given by

$$
\begin{aligned}
\boldsymbol{\zeta}(k+1) & =\boldsymbol{A}_{d}(k) \boldsymbol{\zeta}(k)+\boldsymbol{b}_{d}(k) \boldsymbol{u}(k) \text { with } \\
\boldsymbol{A}_{d}(k+p) & =\boldsymbol{A}_{d}(k)=\partial \overline{\boldsymbol{x}}^{\star}\left(\bar{q}_{k+2}^{\star}\right) / \partial \overline{\boldsymbol{x}}^{\star}\left(\bar{q}_{k+1}^{\star}\right) \\
\boldsymbol{b}_{d}(k+p) & =\boldsymbol{b}_{d}(k)=\boldsymbol{A}_{d}(k) \boldsymbol{f}\left(\overline{\boldsymbol{x}}^{\star}\left(\bar{q}_{k+1}^{\star}\right), \bar{q}_{k}^{\star}\right)
\end{aligned}
$$

under a p-periodic state feedback

$$
\boldsymbol{u}(k)=-\boldsymbol{k}^{\mathrm{T}}(k) \boldsymbol{\zeta}(k) \text { with } \boldsymbol{k}^{\mathrm{T}}(k+p)=\boldsymbol{k}^{\mathrm{T}}(k)
$$

is equivalent to the periodic system (13), iff for all $k$ the following is true:

$$
\begin{aligned}
& \text { 1. } \alpha(k)=\boldsymbol{c}^{\mathrm{T}}\left(\bar{q}_{k}^{\star}\right) \boldsymbol{f}\left(\overline{\boldsymbol{x}}^{\star}\left(\bar{q}_{k+1}^{\star}\right), \bar{q}_{k}^{\star}\right) \neq 0 \\
& \text { 2. } \boldsymbol{k}^{\mathrm{T}}(k)=\boldsymbol{c}^{\mathrm{T}}\left(\bar{q}_{k}^{\star}\right) / \alpha(k) \\
& \text { 3. } \operatorname{det}\left(\boldsymbol{A}_{d}(k)-\boldsymbol{b}_{d}(k) \boldsymbol{k}^{\mathrm{T}}(k)\right)=0 .
\end{aligned}
$$

Proof. First, define the scaled normal vectors

$$
\tilde{\boldsymbol{c}}^{\mathrm{T}}\left(\bar{q}_{k}^{\star}\right)=\boldsymbol{c}^{\mathrm{T}}\left(\bar{q}_{k}^{\star}\right) / \alpha(k),
$$


which is only feasible, if (21) holds. Then applying the periodic equivalence transformation

$$
\delta \overline{\boldsymbol{x}}(k)=\boldsymbol{T}(k) \boldsymbol{\zeta}(k), \quad \boldsymbol{T}(k)=\left(\partial \overline{\boldsymbol{x}}^{\star}\left(\bar{q}_{k+1}^{\star}\right) / \partial \overline{\boldsymbol{x}}^{\star}\left(\bar{q}_{k}^{\star}\right)\right)^{-1}
$$

to the linearized return map (13) and considering (11) and (24) yields

$$
\boldsymbol{\zeta}(c+1)=\prod_{k=0}^{p-1}\left(\frac{\partial \overline{\boldsymbol{x}}^{\star}\left(\bar{q}_{k+2}^{\star}\right)}{\partial \overline{\boldsymbol{x}}^{\star}\left(\bar{q}_{k+1}^{\star}\right)}-\frac{\partial \overline{\boldsymbol{x}}^{\star}\left(\bar{q}_{k+2}^{\star}\right)}{\partial \overline{\boldsymbol{x}}^{\star}\left(\bar{q}_{k+1}^{\star}\right)} \boldsymbol{f}\left(\overline{\boldsymbol{x}}^{\star}\left(\bar{q}_{k+1}^{\star}\right), \bar{q}_{k}^{\star}\right) \tilde{\boldsymbol{c}}^{\mathrm{T}}\left(\bar{q}_{k}^{\star}\right)\right) \boldsymbol{\zeta}(c) .
$$

To assure the identity of the loop (17), (20) and (25), $\boldsymbol{A}_{d}(k), \boldsymbol{b}_{d}(k)$ and $\boldsymbol{k}^{\mathrm{T}}(k)$ must be equal to the expressions (18), (19) and (22). Moreover, the state transition matrix $\partial \overline{\boldsymbol{x}}^{\star}\left(\bar{q}_{k+2}^{\star}\right) / \partial \overline{\boldsymbol{x}}^{\star}\left(\bar{q}_{k+1}^{\star}\right)$ is regular and $\tilde{\boldsymbol{c}}^{\mathrm{T}}\left(\bar{q}_{k}^{\star}\right) \boldsymbol{f}\left(\overline{\boldsymbol{x}}^{\star}\left(\bar{q}_{k+1}^{\star}\right), \bar{q}_{k}^{\star}\right)=1$ holds. Hence, by Lemma 1 the last constraint (23) must be satisfied as well.

From (22) it follows that $\boldsymbol{k}^{\mathrm{T}}(k)$ and $\boldsymbol{c}^{\mathrm{T}}\left(\bar{q}_{k}^{\star}\right)$ are colinear vectors, which can be directly exploited for solving Problem 1.

Theorem 2. By defining the event function (3) as

$$
\Phi\left(\boldsymbol{x}(t), \bar{q}_{k}^{\star}\right)=\boldsymbol{k}^{\mathrm{T}}(k)\left(\boldsymbol{x}(t)-\overline{\boldsymbol{x}}^{\star}\left(\bar{q}_{k+1}^{\star}\right)\right),
$$

where the periodic state feedback gain $\boldsymbol{k}^{\mathrm{T}}(k)$ stabilizes the equivalent periodic system $\Sigma$ and (26) satisfies the condition (7), local orbital stability of the limit cycle $\mathcal{L}_{\mathrm{LC}}$ is guaranteed.

Proof. Theorem 1 states that the eigenvalues

$$
\lambda_{p, i}\left(\boldsymbol{\Psi}_{\mathrm{cl}}(p, 0)\right)=\lambda_{p, i}\left(\prod_{k=0}^{p-1}\left(\boldsymbol{A}_{d}(k)-\boldsymbol{b}_{d}(k) \boldsymbol{k}^{\mathrm{T}}(k)\right)\right)
$$

of the closed-loop monodromy matrix $\boldsymbol{\Psi}_{\mathrm{cl}}(p, 0)$ of system (17)-(20) are identical to the characteristic multipliers $m_{i}$ of (14). Hence, if these eigenvalues satisfy the condition $\left|\lambda_{p, i}\right|<1$, then all $m_{i}$ are stable as well. Moreover, the event function (26) vanishes at all switch points $\overline{\boldsymbol{x}}^{\star}\left(\bar{q}_{k+1}^{\star}\right)$ of the limit cycle, which under the assumption (7) guarantees that any trajectory $\boldsymbol{x}(t)$ starting in a local neighborhood of $\mathcal{L}_{\mathrm{LC}}$ actually converges to $\mathcal{L}_{\mathrm{LC}}$. The constraints (10) are trivially satisfied because (15) holds for all $\bar{q}_{k}^{\star}$.

By Theorems 1 and 2, the event generator design amounts to solving a constrained pole placement problem for the periodic discrete-time linear system (17)-(19). For the unconstrained problem, a well developed theory exists. How to integrate the constraint sets (7) and (23) in the design procedure is explained in Sect. 4.4. Besides ensuring stability, the equivalence stated in Theorem 1 allows a goal-oriented shaping of the local loop behavior, if the target eigenvalues $\lambda_{p, i}^{d}$ are specified appropriately. 


\subsection{Local stabilizability along a limit cycle}

A solution to the control problem only exists if the DCCS is locally stabilizable along the orbit $\mathcal{L}_{\mathrm{LC}}$. A definition of this property on the basis of the concept of local controllability along a trajectory [21] is given here.

Definition 1. Let $\mathcal{B}_{\delta}\left(\mathcal{L}_{\mathrm{LC}}\right)=\left\{\boldsymbol{x}: \operatorname{dist}\left(\boldsymbol{x}, \mathcal{L}_{\mathrm{LC}}\right)<\delta\right\}, \delta>0$ define a local neighborhood of the limit cycle $\mathcal{L}_{\mathrm{LC}}$. A state $\boldsymbol{x}_{0} \in \mathcal{B}_{\delta}\left(\mathcal{L}_{\mathrm{LC}}\right)$ is locally stabilizable along the orbit $\mathcal{L}_{\mathrm{LC}}$, if there exists an $\epsilon>0$, a mode sequence $q(t)$ and a trigger signal $\mathrm{clk}(t)$, such that the following holds:

$$
\begin{aligned}
& \text { 1. } \boldsymbol{x}\left(t, \boldsymbol{x}_{0}, q(0)\right) \in \mathcal{B}_{\epsilon}\left(\mathcal{L}_{\mathrm{LC}}\right), \quad \forall t>0 \\
& \text { 2. } \lim _{t \rightarrow \infty} \operatorname{dist}\left(\boldsymbol{x}\left(t, \boldsymbol{x}_{0}, q(0)\right), \mathcal{L}_{\mathrm{LC}}\right)=0 .
\end{aligned}
$$

Definition 2. A periodically operated DCCS is called locally stabilizable along the orbit $\mathcal{L}_{\mathrm{LC}}$, if all states $\boldsymbol{x}_{0}$ in an open neighborhood $\mathcal{B}_{\delta}\left(\mathcal{L}_{\mathrm{LC}}\right), \delta>0$ of $\mathcal{L}_{\mathrm{LC}}$ are locally stabilizable.

From the results of Theorems 1 and 2 a sufficient condition immediately follows, which ensures local stabilizability along a limit cycle for a DCCS.

Theorem 3. A periodically operated DCCS (2), (5) is locally stabilizable along the orbit $\mathcal{L}_{\mathrm{LC}}$, if the equivalent system $\Sigma=\left\{\boldsymbol{A}_{d}(k), \boldsymbol{b}_{d}(k)\right\}$ is stabilizable.

Proof. To prove Theorem 3, pick any mode $\bar{q}_{k}^{\star} \in \mathcal{Q}$ and assume that the equivalent periodic system $\Sigma$ is stabilizable. Then by Theorems 1 and 2 , stabilizing gains $\boldsymbol{k}^{\mathrm{T}}(k)$ exists, which translate into stabilizing normal directions $\boldsymbol{c}^{\mathrm{T}}\left(\bar{q}_{k}^{\star}\right)$. As the return map $\boldsymbol{P}_{x}$ is a local $C^{1}$ diffeomorphism, there exists a non-empty invariant open region $\mathcal{B}_{\gamma}\left(\overline{\boldsymbol{x}}^{\star}\left(\bar{q}_{k}^{\star}\right)\right)=\left\{\boldsymbol{x} \mid\left(\boldsymbol{x}-\overline{\boldsymbol{x}}^{\star}\left(\bar{q}_{k}^{\star}\right)\right)^{\mathrm{T}} \boldsymbol{V}\left(\boldsymbol{x}-\overline{\boldsymbol{x}}^{\star}\left(\bar{q}_{k}^{\star}\right)\right)<\gamma\right\}$ with $\boldsymbol{V}, \gamma>0$ in the plane $\mathcal{S}\left(\bar{q}_{k}^{\star}\right)$, for which all trajectories that emanate from $\mathcal{B}_{\gamma}\left(\overline{\boldsymbol{x}}^{\star}\left(\bar{q}_{k}^{\star}\right)\right)$ asymptotically converge to the limit cycle. The backward reachable set of $\mathcal{B}_{\gamma}\left(\overline{\boldsymbol{x}}^{\star}\left(\bar{q}_{k}^{\star}\right)\right)$ then defines a non-empty neighborhood of stabilizable states along $\mathcal{L}_{\mathrm{LC}}$, from which the values $\epsilon>0$ and $\delta>0$ can be extracted (Fig. 2). Now, for all $\boldsymbol{x}_{0} \in \mathcal{B}_{\delta}\left(\mathcal{L}_{\mathrm{LC}}\right)$ the event generator equipped with the event function (26) generates a trigger signal clk $(t)$, such that $\boldsymbol{x}\left(t, \boldsymbol{x}_{0}, q(0)\right)$ converges towards $\mathcal{L}_{\mathrm{LC}}$. Therefore, stabilizability of $\Sigma$ is sufficient for the local stabilizability of the underlying DCCS along $\mathcal{L}_{\mathrm{LC}}$.

Stabilizability of periodic systems is defined in [22] and can be checked numerically as described in [23].

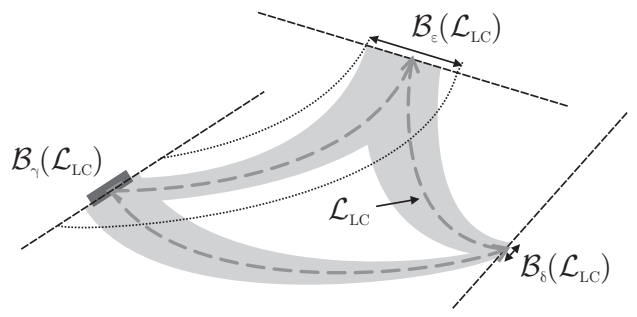

Fig. 2. Limit cycle $\mathcal{L}_{\mathrm{LC}}$, backward reachable set of $\mathcal{B}_{\gamma}\left(\mathcal{L}_{\mathrm{LC}}\right)$ and neighborhoods $\mathcal{B}_{\delta}\left(\mathcal{L}_{\mathrm{LC}}\right), \mathcal{B}_{\epsilon}\left(\mathcal{L}_{\mathrm{LC}}\right)$ defining the region of locally stabilizable states. 


\subsection{Local effectivity of operation modes}

Regarding the design task, it is crucial to identify all effective mode transitions, which can be employed for altering the continuous evolution.

Definition 3. A mode $\bar{q}_{k}^{\star} \in \mathcal{Q}$ of a periodically operated DCCS is called locally effective with respect to the limit cycle $\mathcal{L}_{\mathrm{LC}}$, if it is deactivated upon a controlled switching and a perturbation of $\bar{\tau}^{\star}\left(\bar{q}_{k}^{\star}\right)$ affects the future run of $\boldsymbol{x}(t)$.

All modes $\bar{q}_{k}^{\star}$, which do not meet these two properties, are called ineffective. Modes that are deactivated by an autonomous switching are ineffective, since the corresponding switching conditions must not be altered in the design process. As a result of the above, the periodic sequence $Q_{\mathrm{LC}}=Q_{\mathrm{LC}}^{\mathrm{eff}, 1} Q_{\mathrm{LC}}^{\mathrm{ineff}, 1} \ldots Q_{\mathrm{LC}}^{\mathrm{eff}, j}$ can be decomposed into effective and ineffective subsequences.

Proposition 1. A mode $\bar{q}_{k}^{\star}$ is locally effective, if it is deactivated by a controlled switching and the mode transition causes a discontinuity in the vector field:

$$
\boldsymbol{f}\left(\overline{\boldsymbol{x}}^{\star}\left(\bar{q}_{k+1}^{\star}\right), \bar{q}_{k}^{\star}\right) \neq \boldsymbol{f}\left(\overline{\boldsymbol{x}}^{\star}\left(\bar{q}_{k+1}^{\star}\right), \bar{q}_{k+1}^{\star}\right) .
$$

Proof. While the first part of the proposition is clear, the last part can be proved by contradiction. Assume that (27) is violated. Then, concatenating the linearized embedded map (11) over two consecutively activated modes $\bar{q}_{k}^{\star}$ and $\bar{q}_{k+1}^{\star}$ yields the expression

$$
\delta \overline{\boldsymbol{x}}(k+2)=\left(\boldsymbol{I}-\frac{\boldsymbol{f}\left(\overline{\boldsymbol{x}}^{\star}\left(\bar{q}_{k+2}^{\star}\right), \bar{q}_{k+1}^{\star}\right) \boldsymbol{c}^{\mathrm{T}}\left(\bar{q}_{k+1}^{\star}\right)}{\boldsymbol{c}^{\mathrm{T}}\left(\bar{q}_{k+1}^{\star}\right) \boldsymbol{f}\left(\overline{\boldsymbol{x}}^{\star}\left(\bar{q}_{k+2}^{\star}\right), \bar{q}_{k+1}^{\star}\right)}\right) \frac{\partial \overline{\boldsymbol{x}}^{\star}\left(\bar{q}_{k+2}^{\star}\right)}{\partial \overline{\boldsymbol{x}}^{\star}\left(\bar{q}_{k+1}^{\star}\right)} \frac{\partial \overline{\boldsymbol{x}}^{\star}\left(\bar{q}_{k+1}^{\star}\right)}{\partial \overline{\boldsymbol{x}}^{\star}\left(\bar{q}_{k}^{\star}\right)} \delta \overline{\boldsymbol{x}}(k)
$$

and reveals that $\delta \overline{\boldsymbol{x}}(k+2)$ is independent of $\boldsymbol{c}^{\mathrm{T}}\left(\bar{q}_{k}^{\star}\right)$. Therefore, the mode $\bar{q}_{k}^{\star}$ is ineffective with respect to the control task.

Identifying all ineffective modes prior to performing the design is crucial, as ineffective modes lead to infeasible design results, which include switching planes that violate assumption (10). To reduce computational effort, locally ineffective subsequences $Q_{\mathrm{LC}}^{\text {ineff }, j}$ may be condensed into single modes.

\subsection{Design Algorithm}

Design procedure for $\boldsymbol{Q}_{\mathrm{LC}}=\boldsymbol{Q}_{\mathrm{LC}}^{\mathrm{eff}, 1}$. With the results of Theorems 1 and 2, an event function that guarantees the loop properties specified in Sect. 3 can be obtained as follows:

Algorithm 1 Determination of a stabilizing event function.

Given: a DCCS (2), (5) and an admissible sampled periodic execution $\bar{\chi}^{\star}\left(\boldsymbol{x}_{0}^{h}, 0, p\right)$ evolving on the limit cycle $\mathcal{L}_{\mathrm{LC}}$ to be stabilized.

1. Compute the periodic matrices $\boldsymbol{A}_{d}(k), \boldsymbol{b}_{d}(k)$ according to (18), (19).

2. Verify stabilizability of the equivalent periodic system $\Sigma=\left\{\boldsymbol{A}_{d}(k), \boldsymbol{b}_{d}(k)\right\}$. 
3. Apply periodic pole placement [24] to obtain a periodic feedback gain $\boldsymbol{k}^{\mathrm{T}}(k)$ that simultaneously satisfies the conditions (23) and places the eigenvalues at desired locations $m_{i}^{d}$ in the complex plane.

4. According to (26), set the event function coefficients to $\boldsymbol{c}^{\mathrm{T}}\left(\bar{q}_{k}^{\star}\right)=\boldsymbol{k}^{\mathrm{T}}(k) /\left\|\boldsymbol{k}^{\mathrm{T}}(k)\right\|$ and compute $d\left(\bar{q}_{k}^{\star}\right)=\boldsymbol{c}^{\mathrm{T}}(q) \overline{\boldsymbol{x}}^{\star}\left(\bar{q}_{k+1}^{\star}\right)$.

5. Verify the constraints (7), otherwise introduce additional "counter modes".

Result: Event generator (3), that guarantees local orbital stability of the limit cycle $\mathcal{L}_{\mathrm{LC}}$ and enforces a desired local transient behavior.

The scaling of the normal vectors in Step 4 is admissible, because the Jacobian $\mathrm{d} \boldsymbol{P}_{x} / \mathrm{d} t$ is independent of the length of $\boldsymbol{c}^{\mathrm{T}}\left(\bar{q}_{k}^{\star}\right)$. If stabilizability of (17) is given, the existences of a periodic state feedback $\boldsymbol{k}^{\mathrm{T}}(k)$ that stabilizes the equivalent periodic system is assured for the unconstrained control problem. How to cope with the constraints introduced earlier is explained next.

Handling constraints (23). To explicitly account for the constraints (23) in the design procedure is easy. These conditions only reduce the space of admissible multipliers $m_{i}^{d}$ by one or more dimensions, but a stabilizing solution always exists. The number of multipliers, which must be placed at the origin, depends on the structure of the system (17). It is in general enough to place just one $m_{i}$ at zero, while the remaining ones can be freely assigned in order to shape the transient loop behavior as desired.

Handling constraints (7). Constraints of type (7) are accounted for after the pole assignment. Assume, that Step 5 of Algorithm 1 identifies a mode $\bar{q}_{k}^{\star}$, for which (7) is violated. Then, there exist one or more additional intersections of $\boldsymbol{x}^{\star}\left(\tau, \overline{\boldsymbol{x}}^{\star}\left(\bar{q}_{k}^{\star}\right), \bar{q}_{k}^{\star}\right)$ with $\mathcal{S}\left(\bar{q}_{k}^{\star}\right)$ in the time interval $\left[0, \bar{\tau}^{\star}\left(\bar{q}_{k}^{\star}\right)\right)$. Since the periodic execution $\bar{\chi}^{\star}\left(\boldsymbol{x}_{0}^{h}, 0, p\right)$ requires all mode transitions $\bar{q}_{k}^{\star} \rightarrow \bar{q}_{k+1}^{\star}$ to exactly occur at $\bar{\tau}^{\star}\left(\bar{q}_{k}^{\star}\right)$, the cyclic mode sequence $\mathcal{Q}_{\mathrm{LC}}$ must be extended by auxiliary counter modes $\bar{q}_{k, j}^{\star}$ (Fig. 3). These counter modes are inserted in between $\bar{q}_{k}^{\star}$ and $\bar{q}_{k+1}^{\star}$ and keep track of the number of previous intersections of $\boldsymbol{x}(t)$ and $\mathcal{S}\left(\bar{q}_{k}^{\star}\right)$. They are associated to the same continuous dynamics $\boldsymbol{f}\left(\boldsymbol{x}, \bar{q}_{k, j}^{\star}\right)=\boldsymbol{f}\left(\boldsymbol{x}, \bar{q}_{k}^{\star}\right)$ as $\bar{q}_{k}^{\star}$ and require to augment the event function (3) by

$$
\Phi\left(\boldsymbol{x}, \bar{q}_{k, j}^{\star}\right)=\boldsymbol{c}^{\mathrm{T}}\left(\bar{q}_{k}^{\star}\right) \boldsymbol{x}-d\left(\bar{q}_{k}^{\star}\right)+\epsilon(-1)^{j}, \epsilon \ll d\left(\bar{q}_{k}^{\star}\right) .
$$

Design in the presence of ineffective modes. If a subset of modes is locally ineffective, Algorithm 1 is still applicable. In this case, the system matrices (18)


Fig. 3. Introduction of a counter mode into the cyclic mode sequence, due to violation of the constraint (7). 
and (19) of all ineffective modes become

$$
\begin{aligned}
\boldsymbol{A}_{d}(k+p) & =\boldsymbol{A}_{d}(k)=\partial \overline{\boldsymbol{x}}^{\star}\left(\bar{q}_{k+2}^{\star}\right) / \partial \overline{\boldsymbol{x}}^{\star}\left(\bar{q}_{k+1}^{\star}\right)\left(\boldsymbol{I}-\boldsymbol{f}\left(\bar{q}_{k}^{\star}, \overline{\boldsymbol{x}}^{\star}\left(\bar{q}_{k+1}^{\star}\right)\right) \boldsymbol{c}_{0}^{\mathrm{T}}\left(\bar{q}_{k}^{\star}\right)\right) \\
\boldsymbol{b}_{d}(k+p) & =\boldsymbol{b}_{d}(k)=\mathbf{0} .
\end{aligned}
$$

In this expression, $\boldsymbol{c}_{0}^{\mathrm{T}}\left(\bar{q}_{k}^{\star}\right)$ represents a nominal normal that satisfies the condition $\boldsymbol{c}_{0}^{\mathrm{T}}\left(\bar{q}_{k}^{\star}\right) \boldsymbol{f}\left(\bar{q}_{k}^{\star}, \overline{\boldsymbol{x}}^{\star}\left(\bar{q}_{k+1}^{\star}\right)\right)=1$. Its orientation is either determined from the associated autonomous switching condition or it must be properly chosen, in case $\bar{q}_{k}^{\star}$ is ineffective, because it violates $(27)$.

\section{Experimental validation}

To compare the performance of the novel design approach with earlier results from [16], the new method was successfully applied to the same 2-Tank system consisting of two coupled tanks and a controllable number of inlets and outlets (Fig. 4(a)). For the design, the linear 2-Tank model, its parameters and the desired stationary limit cycle listed in [16] are adopted here again. At stationary operation, the plant recurrently executed the mode sequence $Q_{\mathrm{LC}}=(1234)$. For this sequence, the matrices (18), (19) of the equivalent periodic system $\Sigma$ are

$$
\begin{array}{rlrl}
\boldsymbol{A}_{\mathrm{d}}(1) & =\left(\begin{array}{ll}
0.5572 & 0 \\
0.3544 & 0.6575
\end{array}\right), & \boldsymbol{A}_{\mathrm{d}}(2)=\left(\begin{array}{cc}
0.6421 & 0 \\
0.2693 & 0.5747
\end{array}\right), \\
\boldsymbol{A}_{\mathrm{d}}(3) & =\left(\begin{array}{cc}
0.6534 & 0 \\
0.2637 & 0.5874
\end{array}\right), & \boldsymbol{A}_{\mathrm{d}}(4)=\left(\begin{array}{cc}
0.8004 & 0 \\
0.1839 & 0.8525
\end{array}\right), \\
\boldsymbol{b}_{\mathrm{d}}(1)=(-0.001007-9.117 e-005)^{\mathrm{T}}, & \boldsymbol{b}_{\mathrm{d}}(2)=\left(\begin{array}{ll}
0.0009182 & 0.001224
\end{array}\right)^{\mathrm{T}}, \\
\boldsymbol{b}_{\mathrm{d}}(3)=(0.0006-0.0001484)^{\mathrm{T}}, & \boldsymbol{b}_{\mathrm{d}}(4)=(-0.001808-0.001525)^{\mathrm{T}} .
\end{array}
$$

Since $\Sigma$ turns out to be stabilizable, the underlying DCCS is locally stabilizable along $\mathcal{L}_{\mathrm{LC}}$. It shows that all multipliers $m_{i}$ can be arbitrarily assigned by only using one of the four available feedback gains $\boldsymbol{k}^{\mathrm{T}}(k)$. The large surplus of design parameters is exploited here to maximize the local convergence rate and obtain a dead-beat behavior. Therefore, all desired eigenvalues $\lambda_{i}^{d}$ are set to zero.

The event function coefficients resulting from the application of Algorithm 1 are listed in Tab. 1. They define the switching planes depicted in Figure 4(b) (dashed black lines). The diamonds indicate the switch points $\overline{\boldsymbol{x}}(k)$ of the depicted execution. Note that all switching planes touch the simulated limit cycle $\mathcal{L}_{\mathrm{LC}}$ tangentially (thick grey dashed line), which is a characteristic feature of a dead-beat switching law. For reasons of comparison, the additional thin grey

\begin{tabular}{|c|c|c|c|c|}
\hline$q$ & 1 & 2 & 3 & 4 \\
\hline $\boldsymbol{c}^{\mathrm{T}}(q)$ & $(-0.30960 .9509)^{\mathrm{T}}$ & $(0.78750 .6163)^{\mathrm{T}}$ & $(0.1889-0.9820)^{\mathrm{T}}$ & $(-0.5449-0.8385)^{\mathrm{T}}$ \\
$d(q)$ & 0.005 & 0.0286 & 0.0266 & 0.0058 \\
\hline
\end{tabular}

Table 1. Event function parameters derived from the model-based design procedure. These parameters maximize the convergence rate towards $\mathcal{L}_{\mathrm{LC}}$. 


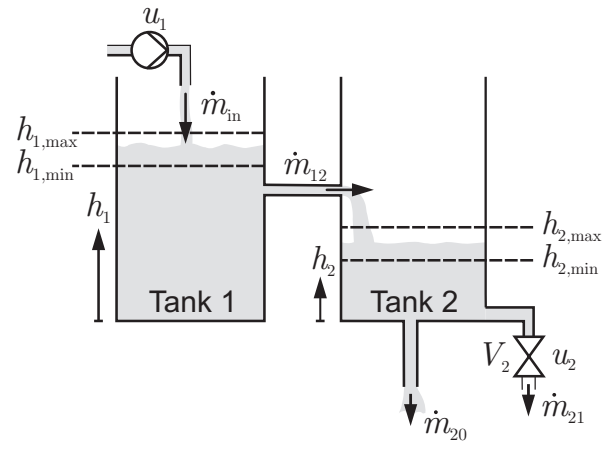

(a)

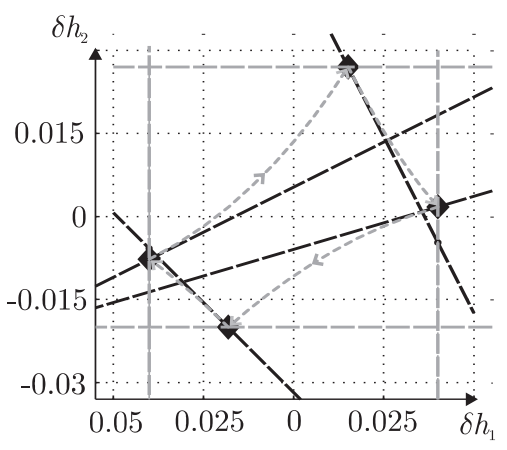

(b)

Fig. 4. 2-Tank system: (a) experimental setup, (b) switching surfaces resulting in local dead-beat behavior.

dashed lines illustrate the paraxial switching planes of the heuristic switching policy explained in [16].

Figure 5 presents a series of state space plots showing experimental data obtained at the laboratory system. They disclose the strong influence of disturbances and model uncertainties, which cause orbital instability when applying the heuristic switching policy. Under the model-based strategy, however, stability is preserved. The experiment was conducted as follows: At runtime, the event function coefficients $\boldsymbol{c}^{\mathrm{T}}\left(\bar{q}_{k}^{\star}\right), d\left(\bar{q}_{k}^{\star}\right)$ were toggled every 1000 seconds between the values listed in Tab. 1 and the values implementing the paraxial planes of the heuristic switching policy. Afterwards, the observed behavior was plotted in a separate figure for each switching policy. For example, the data acquired in the first interval $[0 \mathrm{~s}, 1000 \mathrm{~s}]$ until the substitution of the switching strategy, is plotted in the top-left subplot. Likewise, the behavior observed in the second interval $[1000 \mathrm{~s}, 2000 \mathrm{~s}]$ associated to the heuristic policy is plotted in the top-middle subplot and so on.

As can be concluded from every other subplot, the heuristic switching policy does not succeed in stabilizing $\mathcal{L}_{\mathrm{LC}}$, while the model-based switching policy generates the expected, fast decaying transient loop behavior. The latter is best verified from the subplot "bottom-middle", where the transition onto the limit cycle is finished two switchings after toggling the switching policy. Indeed, the desired periodic operation is preserved even under the influence of considerable disturbances, which is crucial in practical applications.

\section{Conclusion}

The main contribution of this paper is a novel model-based design methodology for the event generator of a periodically operated discretely controlled continuous system. The approach applies to systems of arbitrary state dimension and imposes no restrictions on the number of operation modes. It allows for a goal-oriented shaping of the system's local behavior, in particular to achieve local orbital stability and a fast transient response. Compared to earlier control concepts, the resulting controller issues its actions instantaneously instead of 

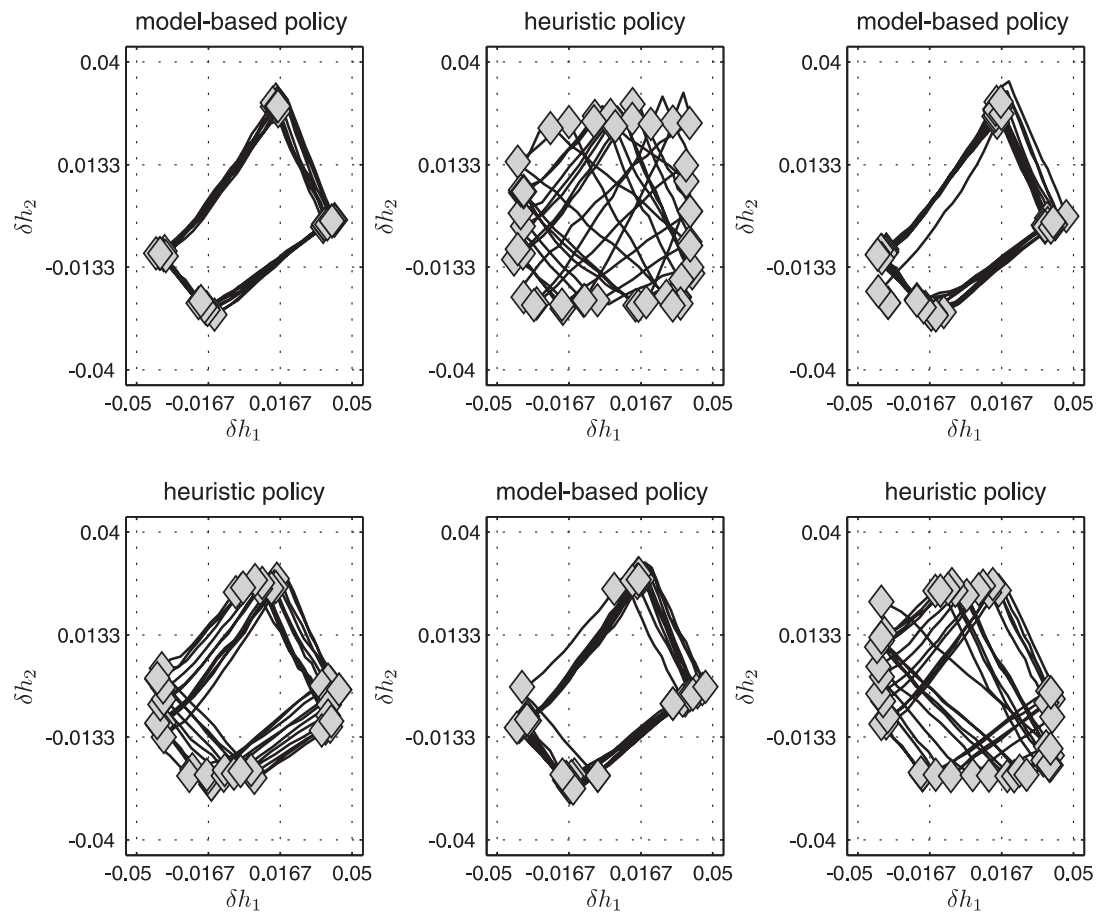

Fig. 5. State space snapshots showing experimental data obtained at the laboratory 2-Tank system. For comparison, the event-generator was toggled between a heuristic and a model-based event-driven policy.

postponing them to the next switching. Thus, the implemented switching strategy guarantees the best possible local loop behavior in the presence of disturbances. As a core feature, the proposed design procedure exploits an equivalence between the original design problem and a classical periodic linear control problem. Based on this idea, the local stabilizability of the DCCS can be investigated. Furthermore, the design is simplified through the application of well known pole placement algorithms for periodic systems.

Future research directions focus on ways for exploiting excessive degrees of freedom to maximize the region of attraction of the stabilized cycle and on concepts for the dynamic adjustment of the switching planes to enable set-point transitions and to compensate for varying parameters. Concerning the method's practical application, it is necessary develop approaches for the design of outputdependent switching laws.

Acknowledgments. This research was supported by the DFG grant LU $462 \mid 21$.

\section{References}

1. di Bernardo, M., Budd, C., Champneys, A., Kowalczyk, P., Nordmark, A., Olivar, G., Piiroinen, P.: Bifurcations in nonsmooth dynamical systems. Technical report, Bristol Centre for Applied Nonlinear Mathematics (2005) 
2. Flieller, D., Riedinger, P., Louis, J.: Computation and stability of limit cycles in hybrid systems. Nonlinear Analysis 64 (2006) 352-367

3. Goncalves, J.: Constructive Global Analysis of Hybrid Systems. PhD thesis, Massachusetts Institute of Technology, Pennsylvania, USA (2000)

4. Hiskens, I.A.: Stability of limit cycles in hybrid systems. In: Proc. of the 34th IEEE Hawaii Int. Conf. on System Sciences, Hawaii (2001)

5. Matveev, A., Savkin, A.: Qualitative Theory of Hybrid Dynamical Systems. Birkhauser (2000)

6. Nordmark, A.: Discontinuity mappings for vector fields with higher order continuity. Dynamical Systems: An International Journal 17(4) (2002) 359-376

7. Rubensson, M., Lennartson, B., Pettersson, S.: Convergence to limit cycles in hybrid systems: An example. In: Proc. IFAC-LSS'98, Rio Patras (1999) 704-709

8. Boccadoro, M., Wardi, Y., Egerstedt, M., Verriest, E.: Optimal control of switching surfaces in hybrid systems. Discrete Event Dynamic Systems 15(4) (2005) 433-448

9. Egerstedt, M., Wardi, Y., Axelsson, H.: Transition-time optimization for switchedmode dynamical systems. IEEE Trans. Autom. Control 51 (2006) 110-115

10. Axelsson, H., Boccadoro, M., Wardi, Y., Egerstedt, M.: Optimal mode-switching for hybrid systems with unknown initial state. In: Proceedings of the 2nd IFAC Conf. on Analysis and Design of Hybrid Systems, Alghero (2006) 95-100

11. Boccadoro, M., Valigi, P., Wardi, Y.: A method for the design of optimal switching surfaces for autonomous hybrid systems. In: Hybrid Systems: Computation and Control. Volume 4416 of LNCS., Pisa, Springer (2007) 650-655

12. Buisson, J., Richard, P.Y., Comerais, H.: On the stabilisation of switching electrical power converters. In: Hybrid Systems: Computation and Control. Number 3414 in LNCS, Zurich, Springer (2005) 185-197

13. Sanders, R., Verghese, G.: Lyapunov-based control for switched power converters. IEEE Transactions on Power Electronics 7 (1992) 17-23

14. Ott, E., Grebogi, C., Yorke, J.: Controlling chaos. Physical Review Letters 64(11) (1990) 1196-1199

15. Dankowicz, H., Piiroinen, P.: Exploiting discontinuities for stabilization of recurrent motions. Dynamical Systems 17(4) (2002) 317-342

16. Schild, A., Lunze, J.: Stabilization of limit cycles of discretely controlled continuous systems by controlling switching surfaces. In: Hybrid Systems: Computation and Control. Volume 4416., Springer (2007) 515-528

17. Krupar, J., Schild, A., Schwarz, W., Lunze, J.: Modeling and analysis of a class of hybrid systems by return maps. In: Proc. of NOLTA, Bologna (2006) 59-62

18. Krupar, J., Lunze, J., Schwarz, W., Schild, A.: Modelling and analysis of discretely controlled continuous systems by means of embedded maps. IEICE Trans. on Fundamentals of Electronics, Communication and Computer Science 89 (2006) 2697-2705

19. Chua, L.O., Parker, T.S.: Practical Numerical Algorithms for Chaotic Systems. Springer Verlag (1989)

20. Bernstein, D.: Matrix Mathematics. Princeton University Press (2007)

21. Sontag, E.D.: Mathematical Control Theory. Springer (1991)

22. Bittanti, S., Bolzern, P.: Stabilizability and detectability of linear periodic systems. Syst. Control Lett. 6(2) (1985) 141-146

23. Sreedhar, J., Dooren, P.V.: An orthogonal method for the controllable subspace of a periodic system. In: Proc. Conf. on Inf. Sciences \& Systems, Baltimore (1993)

24. Sreedhar, J., Dooren, P.V.: Pole placement via the periodic schur decomposition. In: Proc. American Control Conference, San Fransisco (1993) 